\title{
TOWARDS FUZZY GRANULARITY CONTROL IN PARALLEL/DISTRIBUTED COMPUTING
}

\author{
T. Trigo de la Vega, ${ }^{1}$ P. Lopez-García, ${ }^{1,2}$ S. Muñoz-Hernandez ${ }^{3}$ \\ ${ }^{1}$ IMDEA Software, Madrid, Spain \\ ${ }^{2}$ Spanish Research Council (CSIC), Spain \\ ${ }^{3}$ School of Computer Science, Technical University of Madrid (UPM), Spain \\ teresa.trigo@imdea.org,pedro.lopez@imdea.org,susana@fi.upm.es
}

Keywords: $\quad$ Fuzzy Logic Application, Parallel Computing, Automatic Parallelization, Granularity Control, Scheduling, Complexity Analysis.

Abstract: $\quad$ Automatic parallelization has become a mainstream research topic for different reasons. For example, multicore architectures, which are now present even in laptops, have awakened an interest in software tools that can exploit the computing power of parallel processors. Distributed and (multi)agent systems also benefit from techniques and tools for deciding in which locations should processes be run to make a better use of the available resources. Any decision on whether to execute some processes in parallel or sequentially must ensure correctness (i.e., the parallel execution obtains the same results as the sequential), but also has to take into account a number of practical overheads, such as those associated with tasks creation, possible migration of tasks to remote processors, the associated communication overheads, etc. Due to these overheads and if the granularity of parallel tasks, i.e., the "work available" underneath them, is too small, it may happen that the costs are larger than the benefits in their parallel execution. Thus, the aim of granularity control is to change parallel execution to sequential execution or vice-versa based on some conditions related to grain size and overheads. In this work, we have applied fuzzy logic to automatic granularity control in parallel/distributed computing and proposed fuzzy conditions for deciding whether to execute some given tasks in parallel or sequentially. We have compared our proposed fuzzy conditions with existing (conservative) sufficient conditions and our experiments showed that the proposed fuzzy conditions result in more efficient executions on average than the conservative conditions.

\section{INTRODUCTION}

Automatic parallelization is nowadays of great interest since highly parallel processors, which were previously only considered in high performance computing, have steadily made their way into mainstream computing. Currently, even standard desktop and laptop machines include multicore chips with up to twelve cores and the tendency is that these figures will consistently grow in the foreseeable future. Thus, there is an opportunity to build much faster and eventually much better software by producing parallel programs or parallelizing existing ones, and to exploit these new multicore architectures. Performing this by hand will inevitably lead to a decrease in productivity. An ideal alternative is automatic parallelization. There are however some important theoretical and practical issues to be addressed in automatic parallelization. Two of them are: (i) preserving correctness (i.e., ensuring that the parallel execution obtains the same results as the sequential one) and (ii) (theoretical) efficiency (i.e., ensuring that the amount of work performed by executing some tasks in parallel is not greater than the one obtained by executing the tasks sequentially, or at least, there is no slowdown). Solutions to these problems have already been proposed, such as (Chassin and Codognet, 1994; Hermenegildo and Rossi, 1995). However, these solutions assume an idealized execution environment in which a number of practical overheads such as those associated with task creation, possible migration of tasks to remote processors, the associated communication overheads, etc, are ignored. Due to these overheads and if the granularity of parallel tasks, i.e., the "work available" underneath 
them, is too small, it may happen that the costs of parallel execution are larger than its benefits. In order to take these practical issues into account, some methods have been proposed whereby the granularity of parallel tasks and their number are controlled. The aim of granularity control is to change parallel execution to sequential execution or vice-versa based on some conditions related to grain size and overheads. Granularity control has been studied in the context of traditional (Kruatrachue and Lewis, 1988; McGreary and Gill, 1989), functional (Huelsbergen, 1993; Huelsbergen et al., 1994) and logic programming (Kaplan, 1988; Debray et al., 1990; Zhong et al., 1992; LópezGarcía et al., 1996). Taking all these theoretical and practical issues into account, an interesting goal in automatic parallelization is thus to ensure that the parallel execution will not take more time than the sequential one. In general, this condition cannot be determined before executing the task involved, while granularity control should intuitively be carried out ahead of time. Thus, we are forced to use approximations. One clear alternative is to evaluate a (simple) sufficient condition to ensure that the parallel execution will not take more time than the sequential one. This was the approach developed in (López-García et al., 1996). It has the advantage of ensuring that whenever a given group of tasks are executed in parallel, there will be no slowdown with respect to their sequential execution. However, the sufficient conditions can be very conservative in some situations and lead to some tasks being executed sequentially even when their parallel execution would take less time. Although not producing slowdown, this causes a loss in parallelization opportunities, and thus, no speedup is obtained. An alternative is to give up strictly ensuring the no slowdown condition in all parallel executions and to use some conditions that have a good average case behavior. It is in this point where fuzzy logic can be successfully applied to evaluate "fuzzy" conditions that, although can entail eventual slowdowns in some executions, speedup the whole computation on average (always preserving correctness).

It is remarkable the originality of this approach that is betting for the expressiveness of fuzzy logic to improve the decision making in the field of program optimization and, in particular, in automatic program parallelization, including granularity control.

\subsection{Fuzzy Logic Programming}

Fuzzy logic has been a very fertile area during the last years. Specially in the theoretical side, but also from the practical point of view, with the development of many fuzzy approaches. The ones developed in logic programming are specially interesting by their simplicity. The fuzzy logic programming systems replace their inference mechanism, SLD-resolution, with a fuzzy variant that is able to handle partial truth. Most of these systems implement the fuzzy resolution introduced by Lee in (Lee, 1972): the Prolog-Elf system (Ishizuka and Kanai, 1985), the FRIL Prolog system (Baldwin et al., 1995) and the F-Prolog language (Li and Liu, 1990).

One of the most promising fuzzy tools for Prolog was the "Fuzzy Prolog" system (Guadarrama et al., 2004). Fuzzy Prolog adds fuzziness to a Prolog compiler using $\operatorname{CLP}(\mathcal{R})$ instead of implementing a new fuzzy resolution method, as other former fuzzy Prologs do. It represents intervals as constraints over real numbers and aggregation operators as operations with these constraints, so it uses the Prolog built-in inference mechanism to handle the concept of partial truth.

\subsubsection{RFuzzy}

Besides the advantages of Fuzzy Prolog (Vaucheret et al., 2002; Guadarrama et al., 2004), its truth value representation based on constraints is too general, which makes it complex to be interpreted by regular users. That was the reason for implementing a simpler variant that was called RFuzzy (Pablos-Ceruelo et al., 2009a; Muñoz-Hernández et al., 2009; Pablos-Ceruelo et al., 2009b; Strass et al., 2009). In RFuzzy, the truth value is represented by a simple real number.

RFuzzy is implemented as a Ciao Pro$\log$ (Hermenegildo et al., 2008) package because Ciao Prolog offers the possibility of dealing with a higher order compilation through the implementation of Ciao packages.

The compilation process of a RFuzzy program has two pre-compilation steps: (1) the RFuzzy program is translated into CLP $(R)$ constraints by means of the RFuzzy package and (2) the program with constraints is translated into ISO Prolog by using the $\operatorname{CLP}(\mathcal{R})$ package.

As the motivation of RFuzzy was providing a tool for practical application, it was loaded with many nice features that represent an advantage with respect to previous fuzzy tools to model real problems. That is why we have chosen RFuzzy for the implementation of our prototype in this work.

\section{THE GRANULARITY CONTROL PROBLEM}

We start by discussing the basic issues to be addressed in our approach to granularity control, in terms of the 
generic execution model described in (López-García et al., 1996). In particular, we discuss how conditions for deciding between parallel and sequential execution can be devised. We consider a generic execution model: let $g=g_{1}, \ldots, g_{n}$ be a task such that subtasks $g_{1}, \ldots, g_{n}$ are candidates for parallel execution. $T_{S}$ represents the cost (execution time) of the sequential execution of $g$ and $T_{i}$ represents the (sequential) cost of the execution of subtask $g_{i}$.

There can be many different ways to execute $g$ in parallel, involving different choices of scheduling, load balancing, etc., each having its own cost (execution time). To simplify the discussion, we will assume that $T_{p}$ represents in some way all of the possible costs. More concretely, $T_{p} \leq T_{s}$ should be understood as " $T_{s}$ is greater or equal than any possible value for $T_{p}$."

In a first approximation, we assume that the points of parallelization of $g$ are fixed. We also assume, for simplicity, and without loss of generality, that no tests - such as, perhaps, "independence" tests (Chassin and Codognet, 1994; Hermenegildo and Rossi, 1995) other than those related to granularity control are necessary. Thus, the purpose of granularity control will be to determine, based on some conditions, whether the $g_{i}$ 's are going to be executed in parallel or sequentially. In doing this, the objective is to improve the ratio between the parallel and sequential execution times.

Performing an accurate granularity control at compile-time is difficult since most of the information needed, as for example, input data size, is only known at run-time. An useful strategy can be to do as much work as possible at compile-time and postpone some final decisions to run-time. This can be achieved by generating at compile-time cost functions which estimate task costs as a function of input data sizes, which are then evaluated at run-time when such sizes are known. Then, after comparing costs of parallel and sequential executions, it can be determined which of these types of executions must be performed. This scheme was proposed by (Debray et al., 1990) in the context of logic programs and by (Rabhi and Manson, 1990) in the context of functional programs. An interesting goal is to ensure that $T_{p} \leq T_{s}$. In general, this condition cannot be determined before executing $g$, while granularity control should intuitively be carried out ahead of time. Thus, we are forced to use approximations. The way in which these approximations can be performed, is the subject of the two following sections.

\section{THE CONSERVATIVE (SAFE) APPROACH}

The approach proposed in (López-García et al., 1996) consists on using safe approximations, i.e., evaluating a (simple) sufficient condition to ensure that the parallel execution will not take more time than the sequential one. Ensuring $T_{p} \leq T_{S}$ corresponds to the case where the action taken when the condition holds is to run in parallel, i.e., to a philosophy were tasks are executed sequentially unless parallel execution can be shown to be faster. We call this "parallelizing a sequential program." The converse approach, "sequentializing a parallel program," corresponds to the case where the objective is to detect whether the sufficient condition $T_{s} \leq T_{p}$ holds.

Parallelizing a Sequential Program In order to derive a sufficient condition for the inequality $T_{p} \leq T_{S}$, we obtain upper bounds for its left-hand-side and lower bounds for its right-hand-side, i.e., a sufficient condition for $T_{p} \leq T_{s}$ is $T_{p}^{u} \leq T_{s}^{l}$, where $T_{p}^{u}$ denotes an upper bound on $T_{p}$ and $T_{s}^{l}$ a lower bound on $T_{s}$. We will use the superscripts $l$ and $u$ to denote lower and upper bounds respectively throughout the paper. The discussion about how these upper and lower bounds on the sequential and parallel execution times can be estimated are outside the scope of this paper. We refer the reader to (Mera et al., 2008) and (López-García et al., 1996) for a full description of compile-time analysis that obtain lower and upper bounds on sequential and parallel execution times respectively as functions of input data sizes.

Sequentializing a Parallel Program Assume now that we want to detect when $T_{s} \leq T_{p}$ holds, because we have a parallel program and want to profit from performing some sequentializations. In this case, a sufficient condition for $T_{s} \leq T_{p}$ is $T_{s}^{u} \leq T_{p}^{l}$.

\section{THE FUZZY APPROACH}

In some scenarios, it is not allowed to perform parallelizations if it does not ensure any speedup. However, in most environments it is justified to sacrifice efficiency in some cases in order to improve the speedup on average or in the majority of the cases. Thus our approach is to give up strictly ensuring that $T_{p} \leq T_{S}$ holds and to use some relaxed heuristics using fuzzy logic able to detect favorable cases.

We use as a decision criteria the formula $T_{p} \leq T_{s}$. It is easy to transform the formula in $1 \leq T_{s} / T_{p}$ or the 
equivalent $T_{S} / T_{p} \geq 1$. We are implicitly using a crisp criteria in the sense that we use an operator whose truth values are defined mathematically.

If we move to classical logic and want to represent the condition of parallelizing or not a set of subtasks using a logic predicate, we could define greater/2 as a predicate of two arguments that is successful if the first one is greater than the second one and false otherwise. We could check the condition greater $\left(T_{s} / T_{p}, 1\right)$ or rename this condition to a logic predicate, greater $1 / 1$, of arity 1 that compares its argument with 1 , succeeds if it is greater than 1 and fails otherwise (i.e., greaterl(1.8) succeeds, whereas greaterl $(0.8)$ fails). With the boolean condition represented by the predicate greater $1 / 1$ it is easy to follow the conservative approach presented in Section 3.

For a gentle intuition to fuzzy logic, we continue talking about this predicate. We can see that the concept of being "greater than" is very strict in the sense that some cases in which the value is close to 1 are going to be rejected. Let us introduce the concept of truth value. Till now we have been using two truth values true and false, or 1 and 0 . But if we introduce levels of truth we could for example provide for a logic predicate intermediate truth values in between 0 and 1 . We have defined other predicates similar to greaterl/1 that are more flexible in their semantics. They are quite_greater/1 and rather_greater/1. Their definition is clearer in Figure 1 (and described in Section 5.1). With this set of predicates we are going to define a fuzzy framework for the experimental possibilities of using a fuzzy criteria to take decisions about parallelization of tasks.

\subsection{Decision Making}

Instead of deciding about the goodness of the parallelization depending on a crisp condition as in the conservative approach, in this paper we are going to make the decision attending to a couple of certainty factors: $S E Q$, the certainty factor that is going to represent the preference (its truth value) for executing the sequential variant of a program, and $P A R$, the certainty factor that is going to represent the preference (its truth value) for executing the parallel variant of such program. Both certainty factors are real numbers, $S E Q, P A R \in[0,1]$. The way of assigning a value to each certainty factor is not unique. We can define different fuzzy heuristics for their calculation. In Section 5.2 we are going to compare a set of them to choose (in Section 5.3) our selected model.

Once the values of $S E Q$ and $P A R$ have been already assigned, if $P A R>S E Q$ then our task scheduling prototype executes the parallel variant of the program, otherwise it executes the sequential one.

\section{EXPERIMENTAL RESULTS}

We have developed a prototype (Section 5.1) of a fuzzy task scheduler based on the approach described in Section 4. We have prepared a common framework to test the behavior of a set of different heuristics (Section 5.2) and we have compared them also with the rules of the conservative approach (Section 3 ) in order to be able to select the best results (Section 5.3). For a better understanding of these experiments, we present the behavior of our prototype for a progression of execution time data (Section 5.4). Finally, we have tested our prototype with real programs (Section 5.5) in order to demonstrate that it can be successfully applied in practice.

\subsection{Prototype Implementation}

All the granularity control methods have been implemented in Ciao Prolog. The classical logic rules have been implemented using the $\operatorname{CLP}(Q)$ package and the fuzzy logic rules using the Rfuzzy package.

We have decided to use logic programming for implementing our approach because of its simplicity and for taking the advantage of some useful extensions provided by the Ciao Prolog framework. In particular, Ciao Prolog has integrated static analysis techniques for obtaining upper and lower bounds on execution times and a fuzzy library for the calculation of certainty factors.

As explained before, in our new approach to granularity control, the decision of how to execute is based on the certainty factors associated to both, sequential and parallel executions. So that, first of all, we have to quantify such certainty and then decide how to execute. The value to the certainty factors is provided by fuzzy rules that are able to combine fuzzy values using aggregation operators. According to RFuzzy syntax:

$$
\begin{aligned}
& \operatorname{SEQ}\left(P, V_{s}\right): \text { op } \operatorname{cond}_{1}\left(V_{1}\right), \operatorname{cond}_{2}\left(V_{2}\right), \cdots, \operatorname{cond}_{n}\left(V_{n}\right) . \\
& \operatorname{PAR}\left(P, V_{p}\right): \text { op }^{\prime} \operatorname{cond}_{1}^{\prime}\left(V_{1}^{\prime}\right), \operatorname{cond}_{2}^{\prime}\left(V_{2}^{\prime}\right), \cdots, \operatorname{cond}_{n}^{\prime}\left(V_{n}^{\prime}\right) .
\end{aligned}
$$

The truth value $V_{s}$ represents how much executing the program $P$ in a sequential way is adequate. $V_{s}$ is obtained by combining the truth values of the partial conditions $V_{1}, \ldots, V_{n}$ with the aggregation operator $o p$. Symmetrically, $V_{p}$ represents how much adequate is the parallel execution for the program $P$.

The bigger factor (SEQ or PAR) will point out the selected execution (sequential or parallel).

In order to test the behavior of our method we have developed a set of conditions comparing a group 

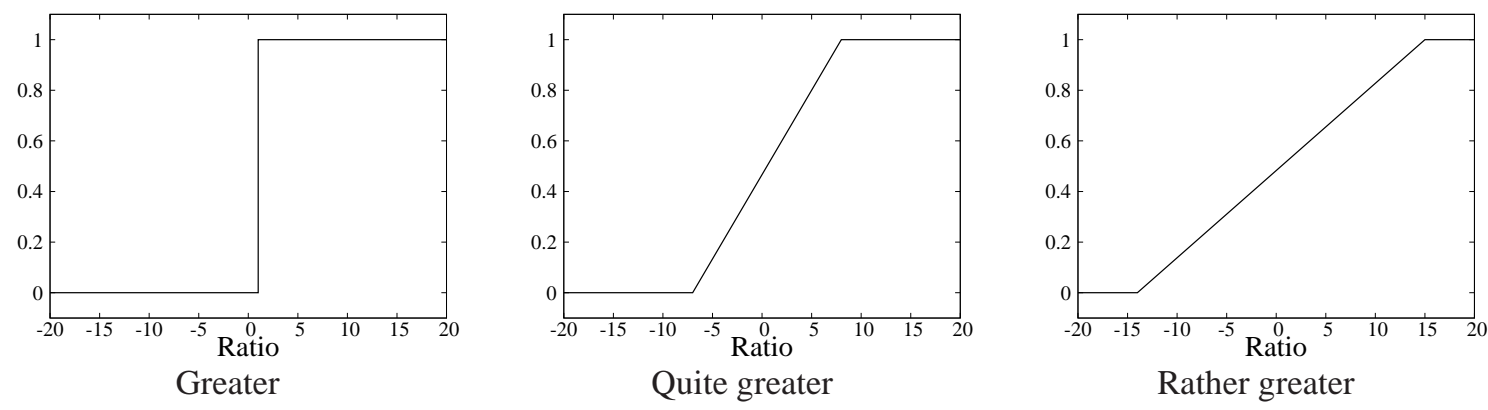

Figure 1: Fuzzy sets for greater.

of values of execution times: $\left\{T_{p}^{l}, T_{p}^{m}, T_{p}^{u}, T_{s}^{l}, T_{s}^{m}, T_{s}^{u}\right\}$ by pairs. The comparison that makes each condition is calculated with the fuzzy relations quite_greater and rather_greater (represented in Figure 1), whose definitions are:

$$
\begin{aligned}
& \text { quite_greater }(X)=\left\{\begin{array}{llc}
0 & \text { if } & X \leq-7 \\
\frac{X+7}{15} & \text { if } & -7<X<8 \\
1 & \text { if } & X \geq 8
\end{array}\right. \\
& \text { rather_greater }(X)=\left\{\begin{array}{llc}
0 & \text { if } & X \leq-14 \\
\frac{X+14}{29} & \text { if } & -14<X<15 \\
1 & \text { if } & X \geq 15
\end{array}\right.
\end{aligned}
$$

We also use the relative harmonic difference, an experimental relation described in (Mera et al., 2008) as follows:

harmonic_diff $(X, Y)=(X-Y) *(1 / X+1 / Y) / 2$. We have selected this relation because it compares two numbers in a relative and symmetric way, i.e.: harmonic_diff $(X, Y)=-$ harmonic_diff $f(Y, X)$. The harmonic difference only works well for positive numbers, but as we are working with execution times, it is enough for our purposes.

These fuzzy relations can be redefined with different bounds, although in this prototype we have only used the values 0,7 and 14. These bounds have been selected according to the magnitude of the execution times that we provide for the programs (see Table 2) in order to obtain significant results depending on the selected fuzzy relation.

\subsection{Heuristic Comparison}

In this section we discuss the evaluation of our prototype with different aggregation operators. A suite of benchmarks to test the prototype has been developed. Each benchmark has been defined in terms of its execution times (average, upper and lower bounds on parallel and sequential execution times) in order to see if the new approach provides better results than the conservative one. Obviously, in real cases, these values will need to be estimated at
Table 1: Aggregation operators execution time.

\begin{tabular}{|c|c|c|c|}
\hline \multirow{2}{*}{ Program } & \multicolumn{3}{|c|}{ Aggregation Operator } \\
\cline { 2 - 4 } & max & dprod & dluka \\
\hline p1 & 1.23 & 1.11 & 1.04 \\
\hline p2 & 0.42 & 0.51 & 0.45 \\
\hline p3 & 0.93 & 0.88 & 0.88 \\
\hline p4 & 0.43 & 0.51 & 0.45 \\
\hline p5 & 0.62 & 0.76 & 0.63 \\
\hline p6 & 0.56 & 0.62 & 0.57 \\
\hline \hline average & 0.70 & 0.73 & 0.67 \\
\hline
\end{tabular}

compile-time using a program analyzer like, for example, CiaoPP (Hermenegildo et al., 2005; Mera et al., 2008). Table 2 contains the description of the benchmarks. Each row shows the information of one program. The first column contains the name of the program and, under it and between brackets, the name of the figure which contains the graphical representation of the benchmark. This figure allows to identify the optimal execution in a graphic way. The following columns show : $T_{s}^{l}$ (lower bound on sequential execution time), $T_{s}^{m}$ (average sequential execution time), $T_{s}^{u}$ (upper bound on sequential execution time), $T_{p}^{l}$ (lower bound on parallel execution time), $T_{p}^{m}$ (average parallel execution time) and $T_{p}^{u}$ (upper bound on parallel execution time). Each execution time is in microseconds.

Figures 2, 3, 4, 5, 6 and 7 describe the benchmarks in a graphic way. In horizontal we find both (parallel and sequential) executions. In vertical we find, for each execution, the interval comprised between its upper and lower bound on execution time.

To make things simpler, we refer to the fuzzy set as $g t$, and to the relative harmonic difference relation as $h d$.

The rules of fuzzy logic for calculating each condition $P A R_{i}$ or $S E Q_{i}, 1 \leq i \leq 7$ (see Table 3), have been composed using several aggregation operators but the 
Table 2: Benchmark program execution times (in microseconds).

\begin{tabular}{|c|c|c|c|c|c|c|}
\hline Program Time & $T_{s}^{l}$ & $T_{s}^{m}$ & $T_{s}^{u}$ & $T_{p}^{l}$ & $T_{p}^{m}$ & $T_{p}^{u}$ \\
\hline \hline $\begin{array}{c}\mathrm{p} 1 \\
\text { (Figure 2) }\end{array}$ & 400 & 600 & 800 & 100 & 175 & 250 \\
\hline $\begin{array}{c}\mathrm{p} 2 \\
\text { (Figure 3) }\end{array}$ & 50 & 175 & 300 & 350 & 550 & 750 \\
\hline $\begin{array}{c}\mathrm{p} 3 \\
\text { (Figure 4) }\end{array}$ & 250 & 525 & 800 & 300 & 375 & 450 \\
\hline $\begin{array}{c}\mathrm{p} 4 \\
\text { (Figure 5) }\end{array}$ & 50 & 150 & 250 & 100 & 325 & 550 \\
\hline $\begin{array}{c}\mathrm{p} 5 \\
\text { (Figure 6) }\end{array}$ & 200 & 400 & 600 & 200 & 325 & 450 \\
\hline $\begin{array}{c}\mathrm{p} 6 \\
\text { (Figure 7) }\end{array}$ & 150 & 325 & 500 & 100 & 275 & 450 \\
\hline
\end{tabular}

results have shown that only the t-conorms max ( $\max$ ), Lukasiewicz (dluka) and sum (dprod) are correct (i.e., always suggest the optimal execution) so we do not show the rest of the tested operations ${ }^{1}$ in the results. We have seen how the three t-conorms max ( $\max$ ), Lukasiewicz (dluka) and sum (dprod) have the same behavior. Thus, in order to chose one of these aggregation operators, we have followed the criteria of the one more efficiently evaluated. In this sense, we have measured the execution time of evaluating the condition $P A R_{1}$ for each program using the three operators. These execution times have been obtained over an Intel platform (Intel Pentium 4 CPU 2.60GHz). They are shown in Table 1. The first column shows the name of the program (see Table 2) and the three next ones, the aggregation operators. Each row shows the execution time (in microseconds) of the evaluation of the condition $P A R_{1}$ (see Table 3 ) for the program using the three mentioned operators. The last row contains, for each operator, an average value on the execution time of evaluating such condition for all the programs. As we can see, the results are very similar for the aggregation operators max and dluka while for dprod are almost always bigger. Although $\max$ is a little bit less efficient (on average) than dluka, max seems to be the best option due to its simplicity.

The whole set of proposed certainty factors and the results for each approach are shown in Table 3. They correspond to the case of parallelizing a sequential program (i.e., where the action taken by default when there is no evidence towards executing is parallel is to execute sequentially). The first column shows the name of the program. The second column shows what would be the right (optimal) decision about the type of execution that should be performed (either pa-

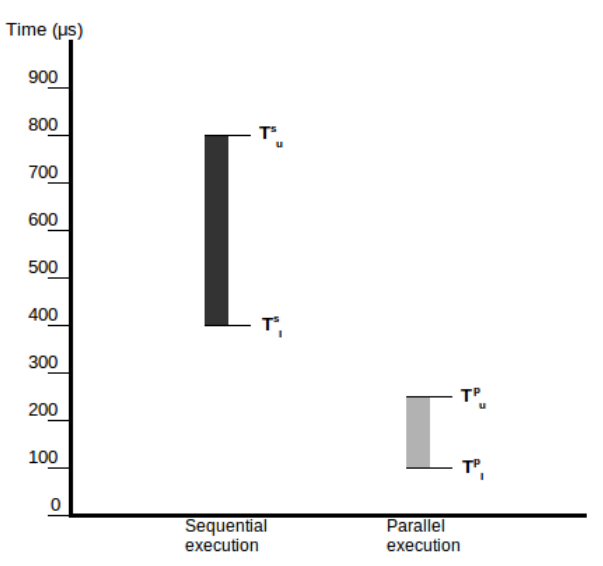

Figure 2: Program p1.

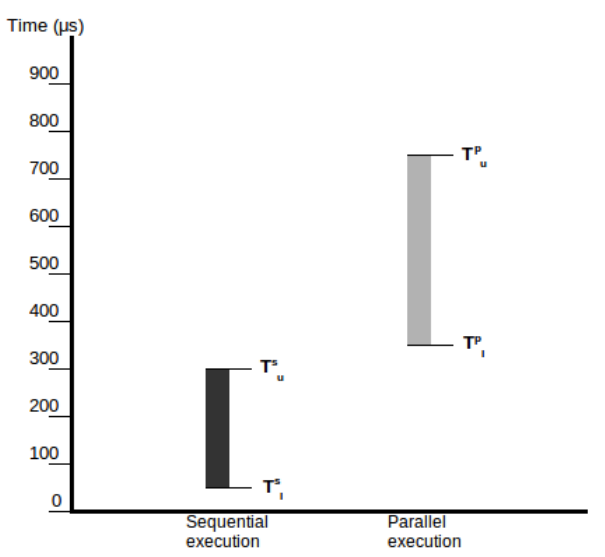

Figure 3: Program p2.

\footnotetext{
${ }^{1}$ The rest of the tested operations are: $\min$, luka and prod.
} 
Table 3: Selected executions using the whole set of rules.

\begin{tabular}{|c|c|c|c|c|c|c|c|c|c|c|c|c|c|c|c|c|c|}
\hline \multirow{3}{*}{ Program } & \multirow{3}{*}{ Optimal } & \multirow{2}{*}{\multicolumn{2}{|c|}{$\begin{array}{c}\begin{array}{c}\text { Classical Logic } \\
\text { (Greater) }\end{array} \\
\text { Classical }\end{array}$}} & \multicolumn{14}{|c|}{$\begin{array}{l}\text { Fuzzy Logic } \\
\text { (Quite greater) }\end{array}$} \\
\hline & & & & \multicolumn{2}{|c|}{ Fuzzy 1} & \multicolumn{2}{|c|}{ Fuzzy 2} & \multicolumn{2}{|c|}{ Fuzzy 3} & \multicolumn{2}{|c|}{ Fuzzy 4} & \multicolumn{2}{|c|}{ Fuzzy 5} & \multicolumn{2}{|c|}{ Fuzzy 6} & \multicolumn{2}{|c|}{ Fuzzy 7} \\
\hline & & $P A R_{c}$ & $S E Q_{c}$ & $P A R_{1}$ & $S E Q_{1}$ & $P A R_{2}$ & $S E Q_{2}$ & $P A R_{3}$ & $\mathrm{SEQ}_{3}$ & $P R_{4}$ & $\mathrm{SEQ}_{4}$ & $P A R_{5}$ & $S E Q_{5}$ & $P A_{6}$ & $S E Q_{6}$ & $P A R_{7}$ & $\mathrm{SEQ}_{7}$ \\
\hline p1 & Parallel & 1 & 0 & 0.73 & 0.48 & 0.73 & 0.48 & 0.73 & 0.48 & 0.57 & 0.35 & 0.57 & 0.35 & 0.57 & 0.35 & 0.57 & 0.36 \\
\hline $\mathrm{p} 2$ & Sequential & 0 & 1 & 0.48 & 0.93 & 0.49 & 0.93 & 0.49 & 0.93 & 0.34 & 0.58 & 0.33 & 0.59 & 0.34 & 0.58 & 0.31 & 0.58 \\
\hline p3 & Parallel & 0 & 0 & 0.56 & 0.54 & 0.58 & 0.54 & 0.58 & 0.54 & 0.48 & 0.44 & 0.48 & 0.44 & 0.48 & 0.44 & 0.47 & 0.44 \\
\hline p4 & Sequential & 0 & 0 & 0.5 & 0.61 & 0.5 & 0.61 & 0.5 & 0.61 & 0.41 & 0.52 & 0.41 & 0.52 & 0.41 & 0.52 & 0.41 & 0.52 \\
\hline p5 & Parallel & 0 & 0 & 0.54 & 0.53 & 0.55 & 0.53 & 0.55 & 0.53 & 0.47 & 0.45 & 0.47 & 0.45 & 0.47 & 0.45 & 0.47 & 0.45 \\
\hline p6 & Parallel & 0 & 0 & 0.56 & 0.52 & 0.56 & 0.52 & 0.56 & 0.52 & 0.48 & 0.45 & 0.48 & 0.45 & 0.48 & 0.45 & 0.48 & 0.45 \\
\hline
\end{tabular}

\begin{tabular}{|c|c|c|c|c|c|c|c|c|c|c|c|c|c|c|c|c|c|}
\hline \multirow{3}{*}{ Program } & \multirow{3}{*}{ Optimal } & \multirow{2}{*}{\multicolumn{2}{|c|}{$\begin{array}{c}\text { Classical Logic } \\
\text { (Greater) }\end{array}$}} & \multicolumn{14}{|c|}{$\begin{array}{c}\text { Fuzzy Logic } \\
\text { (Rather greater) }\end{array}$} \\
\hline & & & & \multicolumn{2}{|c|}{ Fuzzy 1} & \multicolumn{2}{|c|}{ Fuzzy 2} & \multicolumn{2}{|c|}{ Fuzzy 3} & \multicolumn{2}{|c|}{ Fuzzy 4} & \multicolumn{2}{|c|}{ Fuzzy 5} & \multicolumn{2}{|c|}{ Fuzzy 6} & \multicolumn{2}{|c|}{ Fuzzy 7} \\
\hline & & $P A R_{c}$ & $S E Q_{c}$ & $P A R_{1}$ & $S E Q_{1}$ & $P A R_{2}$ & $S E Q_{2}$ & $P R_{3}$ & $S E Q_{3}$ & $\mathrm{PAR}_{4}$ & $\mathrm{SEQ}_{4}$ & $P A R_{5}$ & $S E Q_{5}$ & $P_{A} R_{6}$ & $S E Q_{6}$ & $\mathrm{PAR}_{7}$ & $S E Q_{7}$ \\
\hline p1 & Parallel & 1 & 0 & 0.62 & 0.49 & 0.62 & 0.49 & 0.62 & 0.49 & 0.53 & 0.42 & 0.53 & 0.42 & 0.53 & 0.42 & 0.54 & 0.42 \\
\hline $\mathrm{p} 2$ & Sequential & 0 & 1 & 0.49 & 0.72 & 0.49 & 0.72 & 0.49 & 0.72 & 0.41 & 0.54 & 0.41 & 0.55 & 0.41 & 0.54 & 0.4 & 0.54 \\
\hline p3 & Parallel & 0 & 0 & 0.53 & 0.52 & 0.54 & 0.52 & 0.54 & 0.52 & 0.49 & 0.47 & 0.49 & 0.47 & 0.49 & 0.47 & 0.48 & 0.47 \\
\hline $\mathrm{p} 4$ & Sequential & 0 & 0 & 0.5 & 0.55 & 0.5 & 0.55 & 0.5 & 0.55 & 0.45 & 0.51 & 0.45 & 0.51 & 0.45 & 0.51 & 0.45 & 0.51 \\
\hline p5 & Parallel & 0 & 0 & 0.52 & 0.51 & 0.52 & 0.51 & 0.52 & 0.51 & 0.48 & 0.47 & 0.48 & 0.47 & 0.48 & 0.47 & 0.48 & 0.47 \\
\hline
\end{tabular}

Conditions:

$P A R_{c}$ is $T_{p}^{u} \leq T_{s}^{l}$

$S E Q_{c}$ is $T_{s}^{u} \leq T_{p}^{l}$

$P A R_{1}$ is $\max \left(g t\left(T_{s}^{l} / T_{p}^{u}\right), g t\left(T_{s}^{l} / T_{p}^{l}\right), g t\left(T_{s}^{m} / T_{p}^{m}\right)\right)$

$S E Q_{1}$ is $\max \left(g t\left(T_{p}^{l} / T_{s}^{u}\right), g t\left(T_{p}^{l} / T_{s}^{l}\right), g t\left(T_{p}^{m} / T_{s}^{m}\right)\right)$

$P A R_{2}$ is $\max \left(g t\left(T_{s}^{l} / T_{p}^{u}\right), g t\left(T_{s}^{l} / T_{p}^{l}\right), g t\left(T_{s}^{u} / T_{p}^{u}\right)\right)$

$S E Q_{2}$ is $\max \left(g t\left(T_{p}^{l} / T_{s}^{u}\right), g t\left(T_{p}^{l} / T_{s}^{l}\right), g t\left(T_{p}^{u} / T_{s}^{u}\right)\right)$

$P A R_{3}$ is $\max \left(g t\left(T_{s}^{l} / T_{p}^{u}\right), g t\left(T_{s}^{l} / T_{p}^{l}\right), g t\left(T_{s}^{m} / T_{p}^{m}\right), g t\left(T_{s}^{u} / T_{p}^{u}\right)\right)$

$S E Q_{3}$ is $\max \left(g t\left(T_{p}^{l} / T_{s}^{u}\right), g t\left(T_{p}^{l} / T_{s}^{l}\right), g t\left(T_{p}^{m} / T_{s}^{m}\right), g t\left(T_{p}^{u} / T_{s}^{u}\right)\right)$

$P A R_{4}$ is rel_hd $\left(0.5 * h d\left(T_{s}^{m}, T_{p}^{m}\right)+0.25 * h d\left(T_{s}^{u}, T_{p}^{u}\right)+0.25 * h d\left(T_{s}^{l}, T_{p}^{l}\right)\right)$

$S E Q_{4}$ is rel_hd $\left(0.5 * h d\left(T_{p}^{m}, T_{s}^{m}\right)+0.25 * h d\left(T_{p}^{u}, T_{s}^{u}\right)+0.25 * h d\left(T_{p}^{l}, T_{s}^{l}\right)\right)$

$P A R_{5}$ is rel_hd $\left(\left(h d\left(T_{s}^{m}, T_{p}^{m}\right)+h d\left(T_{s}^{u}, T_{p}^{u}\right)+h d\left(T_{s}^{l}, T_{p}^{l}\right)\right) / 3\right)$

$S E Q_{5}$ is rel_hd $\left(\left(h d\left(T_{p}^{m}, T_{s}^{m}\right)+h d\left(T_{p}^{u}, T_{s}^{u}\right)+h d\left(T_{p}^{l}, T_{s}^{l}\right)\right) / 3\right)$

$P A R_{6}$ is rel_hd $\left(0.25 * h d\left(T_{s}^{m}, T_{p}^{m}\right)+0.5 * h d\left(T_{s}^{u}, T_{p}^{u}\right)+0.25 * h d\left(T_{s}^{l}, T_{p}^{l}\right)\right)$

$S E Q_{6}$ is rel_hd $\left(0.25 * h d\left(T_{p}^{m}, T_{s}^{m}\right)+0.5 * h d\left(T_{p}^{u}, T_{s}^{u}\right)+0.25 * h d\left(T_{p}^{l}, T_{s}^{l}\right)\right)$

$P A R_{7}$ is rel_hd $\left(0.25 * h d\left(T_{s}^{m}, T_{p}^{m}\right)+0.25 * h d\left(T_{s}^{u}, T_{p}^{u}\right)+0.5 * h d\left(T_{s}^{l}, T_{p}^{l}\right)\right)$

$S E Q_{7}$ is rel_hd $\left(0.25 * h d\left(T_{p}^{m}, T_{s}^{m}\right)+0.25 * h d\left(T_{p}^{u}, T_{s}^{u}\right)+0.5 * h d\left(T_{p}^{l}, T_{s}^{l}\right)\right)$ 


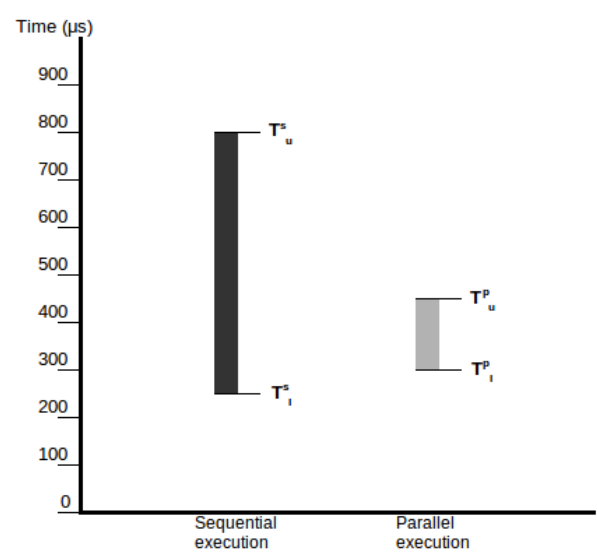

Figure 4: Program p3.

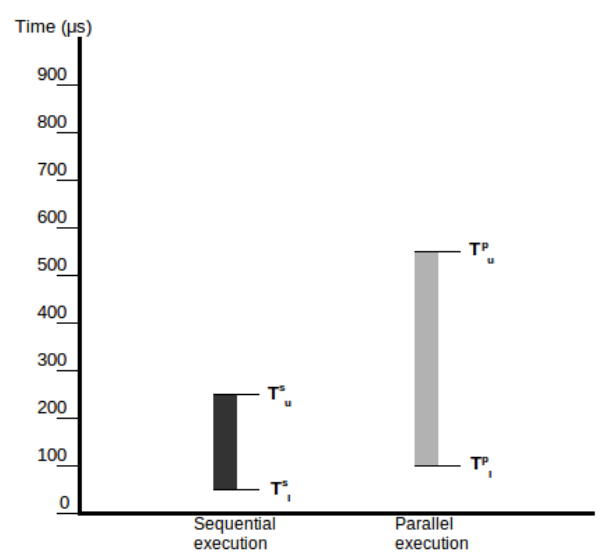

Figure 5: Program p4.

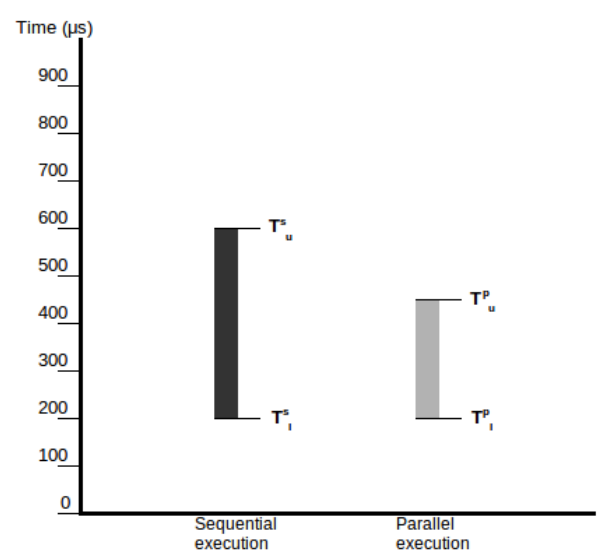

Figure 6: Program p5.

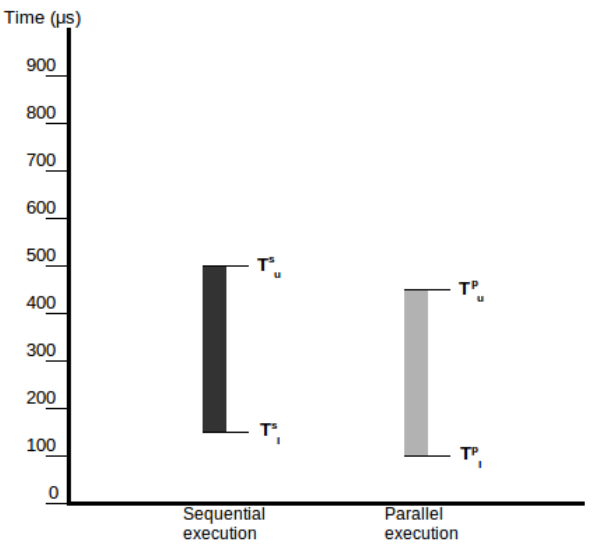

Figure 7: Program p6.

rallel or sequential). The rest of the columns contain the results of evaluating the conditions. Columns 3 and 4 contain the results obtained using the conservative approach, while columns 5-18 contain the results obtained using our proposed conditions based on fuzzy logic. Each column in the later group of columns corresponds to a different fuzzy condition. The selected type of execution (using the process explained in Section 4.1) are highlighted. $S E Q_{i}$ and $P A R_{i}$ are the truth values obtained for the certainty factors of the sequential and parallel executions of the program $p_{i}$. We have performed the experiments for two different levels of flexibility using quite_greater and rather_greater respectively. The decisions made by using the fuzzy conditions are always the optimal ones for these experiments. However, the conservative approach (classical logic) disagrees with the optimal ones in half of the cases. For example, the condition $T_{p}^{u} \leq T_{s}^{l}$ holds for $p 1$ (see Figure 2). Thus, the parallel execution of $p l$ is more efficient than the sequential one. In this case, both the conservative approach (classical logic) and the fuzzy logic approach agree in that the execution of $p l$ should be parallel. The converse condition $\left(T_{s}^{u} \leq T_{p}^{l}\right)$ holds for program $p 2$ (see Figure 3 ), and thus, the optimal action is executing it sequentially. In this case, also both approaches agree in that the execution of $p 2$ should be sequential.

For programs 3-6, the classical logic truth values $\left(P A R_{c}\right.$ and $\left.S E Q_{c}\right)$ are always zero, which means that the suggested type of execution is sequential for all of these programs (i.e., the default type of execution). However, from Figures 4, 5, 6 and 7, we can see that in some cases the optimal decision is to execute these programs in parallel. For example, consider program $p 3$ (see Figure 4). We have that $T_{p}^{u}=450 \mu \mathrm{s}$ and $T_{s}^{l}=250 \mu s$, and thus $T_{p}^{u} \leq T_{s}^{l}$ does not hold. 
The decision of executing p3 sequentially made by classical logic is safe. However, in this case, since $T_{s}^{u}=800 \mu s$, assuming that $p 3$ is run a significant number of times, we have that on average, executing p3 in parallel would be more efficient than executing it sequentially. Unlike the classical logic conditions our proposed fuzzy approach selects the optimal type of execution for p3: its two subtasks should be executed in parallel. Program $p 4$ (see Figure 5) represents the opposite case. In this case $T_{s}^{u}=250 \mu s$ and $T_{p}^{l}=100 \mu s$ so $T_{s}^{u} \leq T_{p}^{l}$ does not hold. But in this case $T_{p}^{u}=550 \mu s$ and $T_{s}^{u}=250 \mu s$. Thus, the best choice seems to be executing $p 4$ sequentially. Using classical logic, the selected execution is sequential, the one selected by default when none of the sufficient conditions $P A R_{c}$ nor $S E Q_{c}$ hold. Using fuzzy logic the selected execution is also sequential. However our conditions provide enough evidences that support the decision of executing sequentially.

In the situations illustrated by the last two programs it is not so clear what type of execution should be selected. For program $p 5$ (see Figure 6) we have that $T_{p}^{u}=450 \mu s$ and $T_{s}^{l}=200 \mu s$. Thus, since the sufficient condition $T_{p}^{u} \leq T_{s}^{l}$ for executing in parallel does not hold, it seems that the program should be executed sequentially. However, since $T_{p}^{l}=200 \mu \mathrm{s}$ and $T_{s}^{u}=600 \mu s$, the sufficient condition $T_{s}^{u} \leq T_{p}^{l}$ for executing in parallel does not hold either. Now, using our fuzzy logic approach, taking the four values $T_{p}^{l}, T_{p}^{u}, T_{s}^{l}$ and $T_{s}^{u}$ into account, a certainty factor of nearly 0.5 suggests that the best choice is to execute p5 in parallel.

For program $p 6$ (see Figure 7), none of the sufficient conditions $T_{p}^{u} \leq T_{s}^{l}$ and $T_{s}^{u} \leq T_{p}^{l}$ (for selecting parallel and sequential execution respectively) hold. However, since $T_{p}^{u} \leq T_{s}^{u}$ and $T_{p}^{l} \leq T_{s}^{l}$ hold, it is clear that the execution time of the sequential execution is going to belong to an interval whose limits are bigger than the limits of the parallel execution. Thus, is it more likely that the execution time of the parallel execution be less than the execution time of the sequential one, so that the right decision seems to execute $p 6$ in parallel. We can see that our proposed fuzzy conditions also suggests the parallel execution.

Finally, we can see that in those cases in which classical logic suggests a type of execution (with truth value 1), our fuzzy logic approach suggests the same type of execution (sequential or parallel).

\subsection{Selected Fuzzy Condition}

Table 3 shows that all the fuzzy conditions (Fuzzy 17) select the same type of execution, sequential or parallel (independently of the fuzzy set used, either quite_greater or rather_greater). Our goal is to detect those situations where the parallel execution is faster than the sequential one, such that a conservative (safe) approach is not able to detect it but the fuzzy approach is. Approaches Fuzzy 4, 5, 6 and 7 suggest parallel execution with less evidence than Fuzzy 1, 2 and 3 for both fuzzy sets (quite_greater and rather_greater). As we are interested in suggesting to execute in parallel with evidences as bigger as possible we rule out this subset of conditions and we focus our attention in the first set. Both Fuzzy 2 and 3 obtain the same values in all cases. Furthermore they provide higher evidences for parallel execution than the condition Fuzzy 1. This fact can be seen in programs $p 3, p 5$ and $p 6$. As Fuzzy 2 is a subset of Fuzzy 3, evaluating the first one is more efficient than the second one (the Fuzzy 3 condition has one more comparison). Thus, the condition that we have selected is Fuzzy 2:

$$
\text { PAR is } \max \left(g t\left(T_{s}^{l} / T_{p}^{u}\right), g t\left(T_{s}^{l} / T_{p}^{l}\right), g t\left(T_{s}^{u} / T_{p}^{u}\right)\right)
$$

This condition obtains a better average case behavior by relaxing decision conditions (and losing some precision). There may be cases in which our approach will select the slowest execution, however it will select the fastest one in a bigger number of cases. This tradeoff between safety and efficiency makes this new approach only applicable to non-critical systems, where no constraints about execution times must be met, and a wrong decision will only cause a slowdown which is admissible. In the same way that it happens in the conservative approach, the fuzzy approach for sequentializing a parallel program is also symmetric to the problem of parallelizing a sequential program. The condition that we have selected for sequentializing a parallel program is:

$$
\text { SEQ is } \max \left(g t\left(T_{p}^{l} / T_{s}^{u}\right), g t\left(T_{p}^{l} / T_{s}^{l}\right), g t\left(T_{p}^{u} / T_{s}^{u}\right)\right)
$$

\subsection{Decisions Progression}

Focusing on program $p 3$ and using the fuzzy set quite_greater with the selected fuzzy model (in Section 5.3) we have developed an incremental experiment whose results are shown in Table 4. The main goal is to see how with this fuzzy logic approach we can select the optimal execution in those cases in which the conservative approach is not able to give a conclusion, and also, how our fuzzy logic approach detects all situations (safely) detected optimal by the conservative approach. Figure 8 shows all the execution scenarios. The sequential execution times are fixed, while the parallel execution ones depend on each scenario. The later are represented by pairs $\left(T_{p}^{l}(i), T_{p}^{u}(i)\right)$ where $i$ is the concrete case. The parallel execution 


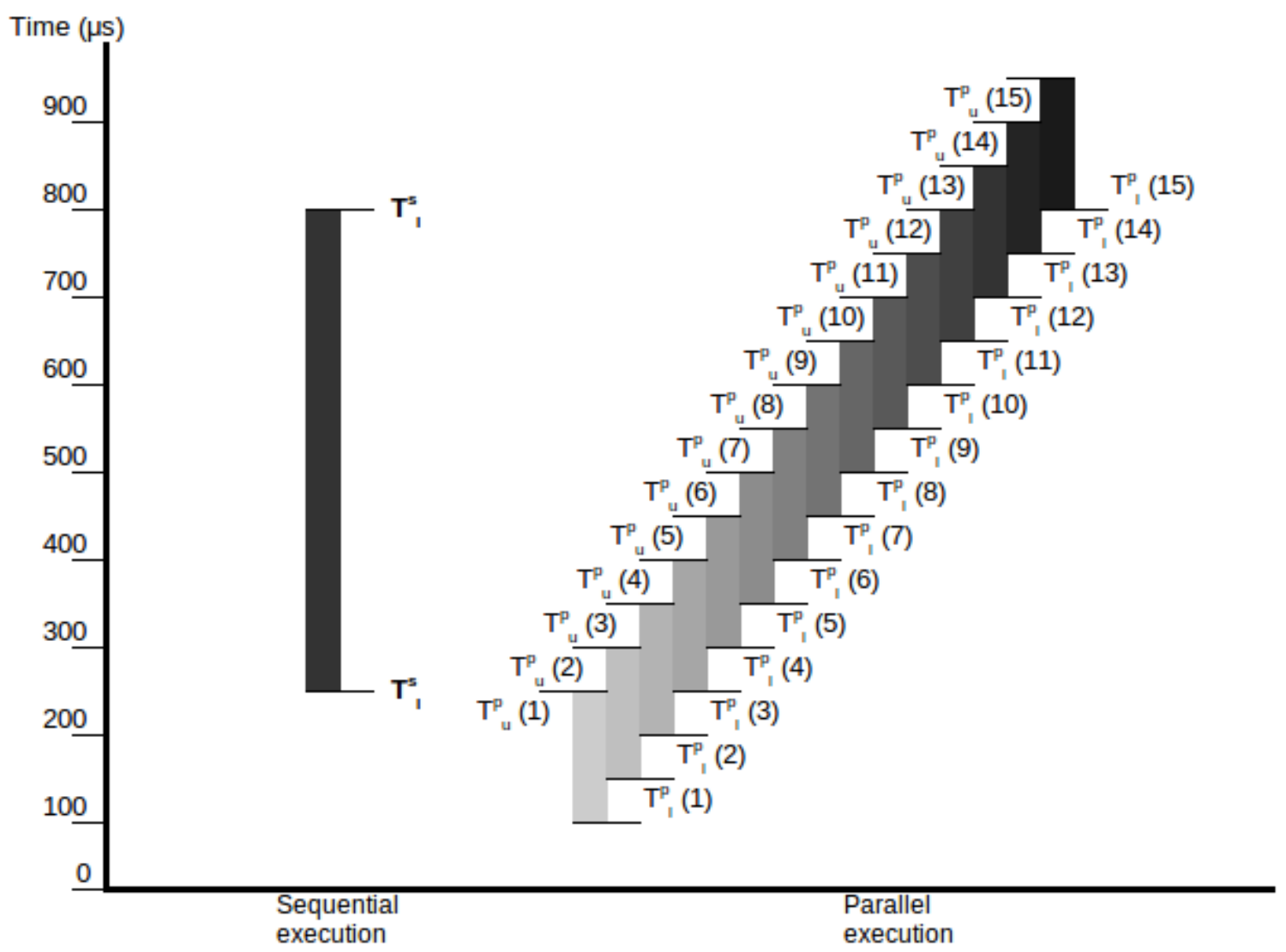

Figure 8: Progression of executions of the example program p3.

times of each scenario are the times of the previous one plus 50 units, in order to appreciate the progression. The times of the first scenario are $T_{p}^{l}(1)=100 \mu \mathrm{s}$ and $T_{p}^{u}(1)=250 \mu s$. Attending to classical logic we can see how only when $P A R_{c}=1$ or $S E Q_{c}=1$ we obtain a justified answer (that the program must be executed in parallel or sequentially respectively). In the rest of the cases the selected type of execution is sequential by default, since we are following the philosophy of parallelizing a sequential program, and there are no evidences towards either type of execution. On the other hand, fuzzy logic always selects the optimal execution (supported by evidences).

\subsection{Experiments with Real Programs}

The former experiments (Section 5.2) have shown that our fuzzy granularity control framework is able to capture which is the optimal type of execution on average. Moreover, in order to ensure that our approach can be applied in practice, we have performed some experiments with real programs (and real execution times). The experimental assessment have been made over an
Table 5: Real programs for experimental assessment.

\begin{tabular}{|ll|}
\hline Qsort & qsort(n) sorts a list of n random elements. \\
Fib & fib(n) obtains the nth Fibonacci number. \\
Hanoi & $\begin{array}{l}\text { hanoi(n) solves Hanoi puzzle with } 3 \text { rods } \\
\text { and n disks. }\end{array}$ \\
\hline
\end{tabular}

UltraSparc-T1, 8 cores x 1GHz (4 threads per core), 8GB of RAM, SunOS 5.10.

We have tested the fuzzy model selected in Section 5.3, so that only upper and lower bounds on (parallel and sequential) execution times were needed. Sequential execution times have been measured directly over the execution platform (executing the worst and best possible cases) while the parallel ones have been estimated.

The number of cores of the processor is denoted as $p$, the number of tasks (candidates for parallel or sequential execution) as $n$, and the relation $\lceil n / p\rceil$ is denoted as $k$. We consider two different overheads of parallel execution: (a) the time needed for creating $n$ 
Table 4: Progression of decisions using the fuzzy set quite greater.

\begin{tabular}{|c|c|c|c|c|c|}
\hline \multirow{2}{*}{ Execution } & \multirow{2}{*}{ Optimal } & \multicolumn{2}{|c|}{$\begin{array}{c}\text { Classical Logic } \\
\text { (Greater) }\end{array}$} & \multicolumn{2}{c|}{$\begin{array}{c}\text { Fuzzy Logic } \\
\text { (Quite greater) }\end{array}$} \\
\cline { 3 - 6 } & & \multicolumn{2}{|c|}{ Classical } & \multicolumn{2}{|c|}{ Fuzzy 2} \\
\cline { 3 - 6 } & & $P A R_{c}$ & $S E Q_{c}$ & $P A R_{2}$ & $S E Q_{2}$ \\
\hline p3_execution1 & Parallel & 1 & 0 & 0.68 & 0.49 \\
\hline p3_execution2 & Parallel & 0 & 0 & 0.64 & 0.5 \\
\hline p3_execution3 & Parallel & 0 & 0 & 0.61 & 0.52 \\
\hline p3_execution4 & Parallel & 0 & 0 & 0.6 & 0.53 \\
\hline p3_execution5 & Parallel & 0 & 0 & 0.58 & 0.54 \\
\hline p3_execution6 & Parallel & 0 & 0 & 0.57 & 0.56 \\
\hline p3_execution7 & Sequential & 0 & 0 & 0.56 & 0.57 \\
\hline p3_execution8 & Sequential & 0 & 0 & 0.55 & 0.58 \\
\hline p3_execution9 & Sequential & 0 & 0 & 0.54 & 0.6 \\
\hline p3_execution10 & Sequential & 0 & 0 & 0.54 & 0.61 \\
\hline p3_execution11 & Sequential & 0 & 0 & 0.53 & 0.62 \\
\hline p3_execution12 & Sequential & 0 & 0 & 0.53 & 0.64 \\
\hline p3_execution13 & Sequential & 0 & 0 & 0.52 & 0.65 \\
\hline p3_execution14 & Sequential & 0 & 0 & 0.52 & 0.66 \\
\hline p3_execution15 & Sequential & 0 & 1 & 0.52 & 0.68 \\
\hline
\end{tabular}

Conditions:

$$
\begin{aligned}
& P A R_{c} \text { is } T_{p}^{u} \leq T_{s}^{l} \\
& S E Q_{c} \text { is } T_{s}^{u} \leq T_{p}^{l} \\
& P A R_{2} \text { is } \max \left(g t\left(T_{s}^{l} / T_{p}^{u}\right), g t\left(T_{s}^{l} / T_{p}^{l}\right), g t\left(T_{s}^{u} / T_{p}^{u}\right)\right) \\
& S E Q_{2} \text { is } \max \left(g t\left(T_{p}^{l} / T_{s}^{u}\right), g t\left(T_{p}^{l} / T_{s}^{l}\right), g t\left(T_{p}^{u} / T_{s}^{u}\right)\right)
\end{aligned}
$$

parallel tasks, called Create( $n$ ), and (b) an upper bound on the time taken from the point in which a parallel subtask $g_{i}$ is created until its execution is started by a processor, denoted as SysOverhead $i$. Both types of overheads have been experimentally measured for the execution platform. For the first one, we have measured directly the time of creating $p$ threads. The second one has been obtained by using the expression $(S / 2)-P$, where $S$ and $P$ are the measured execution times of a program consisting of two perfectly balanced tasks running with one and two threads respectively.

There are different ways of executing a task in parallel depending on the scheduling. The highest parallel execution time will be the one with the worst scheduling (i.e., the one in which the cores are idle as much as possible). Consider a task $g=g_{1}, \ldots, g_{n}$ such that subtasks $g_{1}, \ldots, g_{n}$ are candidates for parallel execution. Assume that $T s_{i}$ represents the cost (execution time) of the execution of subtask $g_{i}$. Assume also that $T s_{1}, T s_{2}, \ldots, T s_{n}$ are in descending order of cost and that an ideal parallel execution environment has no parallel execution overheads. Then, we can estimate $T_{p}^{l}$ and $T_{p}^{u}$ as follows:

$$
T_{p}^{l}=T_{s}^{l} / p
$$

$$
T_{p}^{u}=\text { Create }(p)+\sum_{i=1}^{k}\left(\text { SySoverhead }_{i}+T_{s_{i}}^{u}\right)
$$

Table 6 shows the experimental results. The first four columns show the same information as in Table 3, although in this table Program refers to the benchmarks in Table 5. For space reasons, Table 6 only shows results for a subset of inputs. In particular, $\mathrm{Fi}$ bonacci and Hanoi have been tested with the set of inputs $\{1,18\}$ and $\{1,14\}$ respectively. The assessment of the fuzzy approach proposed in this paper is similar for the whole set of tested inputs. The last row shows the speedup of our fuzzy approach with respect to the conservative approach: speedup $=\frac{T_{c}}{T_{f}}$, where $T_{c}$ is the time of the selected execution using the conservative approach and $T_{f}$ is the time of the selected execution using our fuzzy approach. A positive value of speedup means that the execution selected with our approach is faster than the one selected by the conservative approach.

We can distinguish two main sets of cases in Table 6: one set made up of qsort and the other set made up of $f i b$ and hanoi. In the first set the upper bound on the sequential execution time is different from the 
Table 6: Selected executions for real programs.

\begin{tabular}{|c|c|c|c|c|c|c|}
\hline \multirow{2}{*}{ Execution } & \multirow{2}{*}{ Optimal } & $\begin{array}{c}\text { Classical Logic } \\
\text { (Greater) }\end{array}$ & \multicolumn{2}{c|}{$\begin{array}{c}\text { Fuzzy Logic } \\
\text { (Quite greater) }\end{array}$} & \multirow{2}{*}{ Speedup } \\
\cline { 3 - 6 } & & \multicolumn{2}{|c|}{ Classical } & \multicolumn{2}{|c|}{ Fuzzy 2} & \\
\cline { 3 - 6 } & & $P A R_{c}$ & $S E Q_{c}$ & $P A R_{2}$ & $S E Q_{2}$ & \\
\hline \hline qsort(250) & Parallel & 0 & 0 & 0.6 & 0.53 & 1.66 \\
\hline qsort(500) & Parallel & 0 & 0 & 0.6 & 0.53 & 1.74 \\
\hline qsort(750) & Parallel & 0 & 0 & 0.6 & 0.53 & 1.74 \\
\hline qsort(1000) & Parallel & 0 & 0 & 0.6 & 0.53 & 1.75 \\
\hline qsort(1250) & Parallel & 0 & 0 & 0.6 & 0.53 & 1.71 \\
\hline \hline fib(1) & Sequential & 0 & 0 & 0.53 & 0.53 & 1.0 \\
\hline fib(3) & Sequential & 0 & 0 & 0.6 & 0.56 & 0.82 \\
\hline fib(5) & Parallel & 1 & 0 & 0.6 & 0.52 & 1.0 \\
\hline fib(7) & Parallel & 1 & 0 & 0.6 & 0.51 & 1.0 \\
\hline fib(12) & Parallel & 1 & 0 & 0.6 & 0.5 & 1.0 \\
\hline \hline hanoi(4) & Sequential & 0 & 0 & 0.6 & 0.68 & 1.0 \\
\hline hanoi(5) & Sequential & 0 & 0 & 0.6 & 0.58 & 0.94 \\
\hline hanoi(6) & Parallel & 0 & 0 & 0.6 & 0.53 & 1.28 \\
\hline hanoi(7) & Parallel & 1 & 0 & 0.6 & 0.51 & 1.0 \\
\hline hanoi(8) & Parallel & 1 & 0 & 0.6 & 0.5 & 1.0 \\
\hline
\end{tabular}

Conditions:

$$
\begin{aligned}
& P A R_{c} \text { is } T_{p}^{u} \leq T_{s}^{l} \\
& S E Q_{c} \text { is } T_{s}^{u} \leq T_{p}^{l} \\
& P A R_{2} \text { is } \max \left(g t\left(T_{s}^{l} / T_{p}^{u}\right), g t\left(T_{s}^{l} / T_{p}^{l}\right), g t\left(T_{s}^{u} / T_{p}^{u}\right)\right) \\
& S E Q_{2} \text { is } \max \left(g t\left(T_{p}^{l} / T_{s}^{u}\right), g t\left(T_{p}^{l} / T_{s}^{l}\right), g t\left(T_{p}^{u} / T_{s}^{u}\right)\right)
\end{aligned}
$$

lower bound, while in the second set both bounds are the same. This is understandable, since the execution time for the first set of cases not only depends on the length of the input list, but also on the values of its elements. Thus, for a given list length, there may be different execution times, depending on the actual values of the lists with such length. However, in the second set of cases, the execution time only depends on the size (using the integer value metric) of the input argument, and all executions for the same input data size take the same execution time. Our approach improves provides better average case behaviour than the conservative approach in both cases.

\section{CONCLUSIONS}

We have applied fuzzy logic to the program optimization field, in particular, to automatic granularity control in parallel/distributed computing. We have derived fuzzy conditions for deciding whether to execute some tasks in parallel or sequentially, using information about the cost of tasks and parallel execution overheads.
We have performed an experimental assessment of the fuzzy conditions and identified the ones that have the best average case behavior. We have also compared our proposed fuzzy conditions with existing sufficient (conservative) ones for performing granularity control. Our experiments showed that the proposed fuzzy conditions result in better program optimizations (on average) than the conservative conditions. The conservative approach ensures that execution decisions will never result in a slowdown, but loses some parallelizations opportunities (and thus, no speedup is obtained). In contrast, the fuzzy approach makes a better use of the parallel resources and although fuzzy conditions can produce slowdown for some executions, the whole computation benefits from some speedup on average (always preserving correctness). Of course, the fuzzy approach is applicable in scenarios where the no slowdown property is not needed, as for example video games, text processors, compilers, etc.

Experiments performed with real programs (and real execution times) have demonstrated that our approach can be successfully applied in practice. We intend to perform a more rigorous and broad assessment or our approach, by applying it to large real life pro- 
grams and using fully automatic tools for estimating execution times.

Although a lot of work still remains to be done, the preliminary results are very encouraging and we believe that it is possible to exploit all the potential offered by multicore systems by applying fuzzy logic to automatic program parallelization techniques.

\section{ACKNOWLEDGEMENTS}

This research has been partially funded by the EU 7 th. FP NoE $S$-Cube 215483, FET IST-231620 HATS, MICINN TIN-2008-05624 DOVES and CM project P2009/TIC/1465 PROMETIDOS. Teresa Trigo has been supported by CAM grant CPI/0621/2008.

\section{REFERENCES}

Baldwin, J. F., Martin, T., and Pilsworth, B. (1995). Fril: Fuzzy and Evidential Reasoning in Artificial Intelligence. John Wiley \& Sons.

Chassin, J. and Codognet, P. (1994). Parallel Logic Programming Systems. Computing Surveys, 26(3):295-336.

Debray, S. K., Lin, N.-W., and Hermenegildo, M. (1990). Task Granularity Analysis in Logic Programs. In Proc. of the 1990 ACM Conf. on Programming Language Design and Implementation, pages 174-188. ACM Press.

Guadarrama, S., Muñoz, S., and Vaucheret, C. (2004). Fuzzy Prolog: A new Approach Using Soft Constraints Propagation. Fuzzy Sets and Systems, FSS, 144(1):127-150. ISSN 01650114.

Hermenegildo, M., Puebla, G., Bueno, F., and López-García, P. (2005). Integrated Program Debugging, Verification, and Optimization Using Abstract Interpretation (and The Ciao System Preprocessor). Science of Computer Programming, 58(12):115-140.

Hermenegildo, M. and Rossi, F. (1995). Strict and Non-Strict Independent And-Parallelism in Logic Programs: Correctness, Efficiency, and Compile-Time Conditions. Journal of Logic Programming, 22(1):1-45.

Hermenegildo, M. V., Bueno, F., Carro, M., López, P., Morales, J., and Puebla, G. (2008). An Overview of The Ciao Multiparadigm Language and Program Development Environment and its Design Philosophy. In Festschrift for Ugo Montanari, number 5065 in LNCS, pages 209-237. Springer-Verlag.

Huelsbergen, L. (1993). Dynamic Language Parallelization. Technical Report 1178, Computer Science Dept. Univ. of Wisconsin.

Huelsbergen, L., Larus, J. R., and Aiken, A. (1994). Using RunTime List Sizes to Guide Parallel Thread Creation. In Proc. ACM Conf. on Lisp and Functional Programming.

Ishizuka, M. and Kanai, N. (1985). Prolog-ELF incorporating fuzzy logic. In IJCAI, pages 701-703.
Kaplan, S. (1988). Algorithmic Complexity of Logic Programs. In Logic Programming, Proc. Fifth International Conference and Symposium, (Seattle, Washington), pages 780-793.

Kruatrachue, B. and Lewis, T. (1988). Grain Size Determination for Parallel Processing. IEEE Software.

Lee, R. (1972). Fuzzy logic and the resolution principle. Journal of the Association for Computing Machinery, 19(1):119-129.

Li, D. and Liu, D. (1990). A Fuzzy Prolog Database System. John Wiley \& Sons, New York.

López-García, P., Hermenegildo, M., and Debray, S. K. (1996). A Methodology for Granularity Based Control of Parallelism in Logic Programs. Journal of Symbolic Computation, Special Issue on Parallel Symbolic Computation, 21(4-6):715-734.

McGreary, C. and Gill, H. (1989). Automatic Determination of Grain Size for Efficient Parallel Processing. Communications of the ACM, 32 .

Mera, E., López-García, P., Carro, M., and Hermenegildo, M (2008). Towards Execution Time Estimation in Abstract Machine-Based Languages. In 10th Int'l. ACM SIGPLAN Symposium on Principles and Practice of Declarative Programming (PPDP'08), pages 174-184. ACM Press.

Muñoz-Hernández, S., Pablos-Ceruelo, V., and Strass, H. (2009). Rfuzzy: An expressive simple fuzzy compiler. In IWANN (1), pages 270-277.

Pablos-Ceruelo, V., Muñoz-Hernández, S., and Strass, H. (2009a) Rfuzzy framework. Paper presented at the 18th Workshop on Logic-based Methods in Programming Environments (WLPE2008), CoRR, abs/0903.2188.

Pablos-Ceruelo, V., Strass, H., and Muñoz Hernández, S. (2009b). Rfuzzy-a framework for multi-adjoint fuzzy logic programming. In Fuzzy Information Processing Society, 2009. NAFIPS 2009. Annual Meeting of the North American, pages $1-6$

Rabhi, F. A. and Manson, G. A. (1990). Using Complexity Functions to Control Parallelism in Functional Programs. Res. Rep. CS-90-1, Dept. of Computer Science, Univ. of Sheffield, England.

Strass, H., Muñoz-Hernández, S., and Pablos-Ceruelo, V. (2009). Operational semantics for a fuzzy logic programming system with defaults and constructive answers. In IFSA/EUSFLAT Conf., pages 1827-1832.

Vaucheret, C., Guadarrama, S., and Muñoz, S. (2002). Fuzzy Prolog: A Simple General Implementation using CLP(R). In 9th International Conference on Logic for Programming Artificial Intelligence and Reasoning, Tbilisi, Georgia.

Zhong, X., Tick, E., Duvvuru, S., Hansen, L., Sastry, A., and Sundararajan, R. (1992). Towards an Efficient Compile-Time Granularity Analysis Algorithm. In Proc. of the 1992 International Conference on Fifth Generation Computer Systems, pages 809-816. Institute for New Generation Computer Technology (ICOT). 